\title{
Estimated incidence of normal- pressure hydrocephalus and shunt outcome in patients residing in assisted-living and extended-care facilities
}

\author{
Anthony Marmarou, Ph.D., Harold F. Young, M.D., And Gunes A. Aygok, M.D. \\ Department of Neurosurgery, Virginia Commonwealth University Medical Center, Richmond, Virginia
}

Object. The primary objective of this study was to estimate the prevalence of idiopathic normalpressure hydrocephalus (NPH), both diagnosed and undiagnosed, among residents of assisted-living and extended-care facilities, by using a practical screening tool. A secondary objective was to evaluate prospectively the diagnosis and outcome of surgical treatment in a subset of patients residing in healthcare facilities who were at risk for idiopathic NPH.

Methods. A retrospective chart analysis was performed using the medical records from four nursing homes. The final analysis included 147 patient records. Symptomatology and comorbidity were evaluated, as was the ability to perform activities of daily living. In a subset of 17 patients residing in healthcare facilities, the authors applied a standard idiopathic NPH diagnostic and management protocol and followed up the patients 1 year after treatment.

The estimated incidence of suspected idiopathic NPH among all patients in the retrospective survey ranged from 9 to $14 \%$, depending on the diagnostic criteria used. Among the cohort of 17 patients available for an in-hospital study and 1-year follow up, 11 received shunts and seven of these showed either transient or sustained improvement.

Conclusions. A valid and practical diagnostic method is needed to identify idiopathic NPH accurately before admitting patients to a healthcare facility. Data from a prospective study of 17 patients residing in healthcare facilities indicated that supplementary tests remain predictive of a positive response to shunt insertion but cannot predict whether a favorable outcome will be sustained in a population of patients who have been confined to a wheelchair for a prolonged period of time. This finding supports the notion of a finite window of opportunity for successful treatment of idiopathic NPH and the imperativeness of an early diagnosis.

\section{KEY WoRdS • assisted living • dementia • extended care • normal-pressure hydrocephalus $\bullet$ disease prevalence}

$\mathrm{T}$ HE SYMPTOM TRIAD of gait disturbance, urinary incontinence, and dementia in combination with ventriculomegaly clinically defines idiopathic NPH. Abnormal gait is considered the predominant component of this diagnostic triad, and it is rare for a patient with idiopathic NPH to present without some form of gait disturbance. ${ }^{713}$ The characteristic gait is described as shuffling, wide-based, or ataxic. The incontinence associated with idiopathic NPH is characterized by urgency or frequent

Abbreviations used in this paper: $\mathrm{ADL}=$ activities of daily living; $\mathrm{CSF}=$ cerebrospinal fluid; $\mathrm{CT}=$ computed tomography; $\mathrm{ELD}=\mathrm{ex}-$ tended lumbar drainage; $\mathrm{MR}=$ magnetic resonance; $\mathrm{NPH}=$ normalpressure hydrocephalus; PVI = pressure volume index. urination, and the dementia as faltering in short- or longterm memory. ${ }^{19}$ Although the disease was initially defined by Hakim and Adams ${ }^{13}$ in 1965, its precise origin remains elusive. The absence of a clearly established pathophysiology and the presence of possible confounding coexisting dementias or brain disorders with similar symptom patterns make it difficult to establish the clinical diagnosis of idiopathic NPH., ${ }^{2,720}$ As a result, it is frequently misdiagnosed or undiagnosed, ${ }^{12}$ and thus left untreated.

Normal-pressure hydrocephalus is subdivided into two main categories: idiopathic NPH and secondary NPH. The latter usually is caused by events such as subarachnoid hemorrhage, meningitis, head trauma, or stroke ${ }^{19}$ and can develop in patients of all ages. In contrast, there is no 


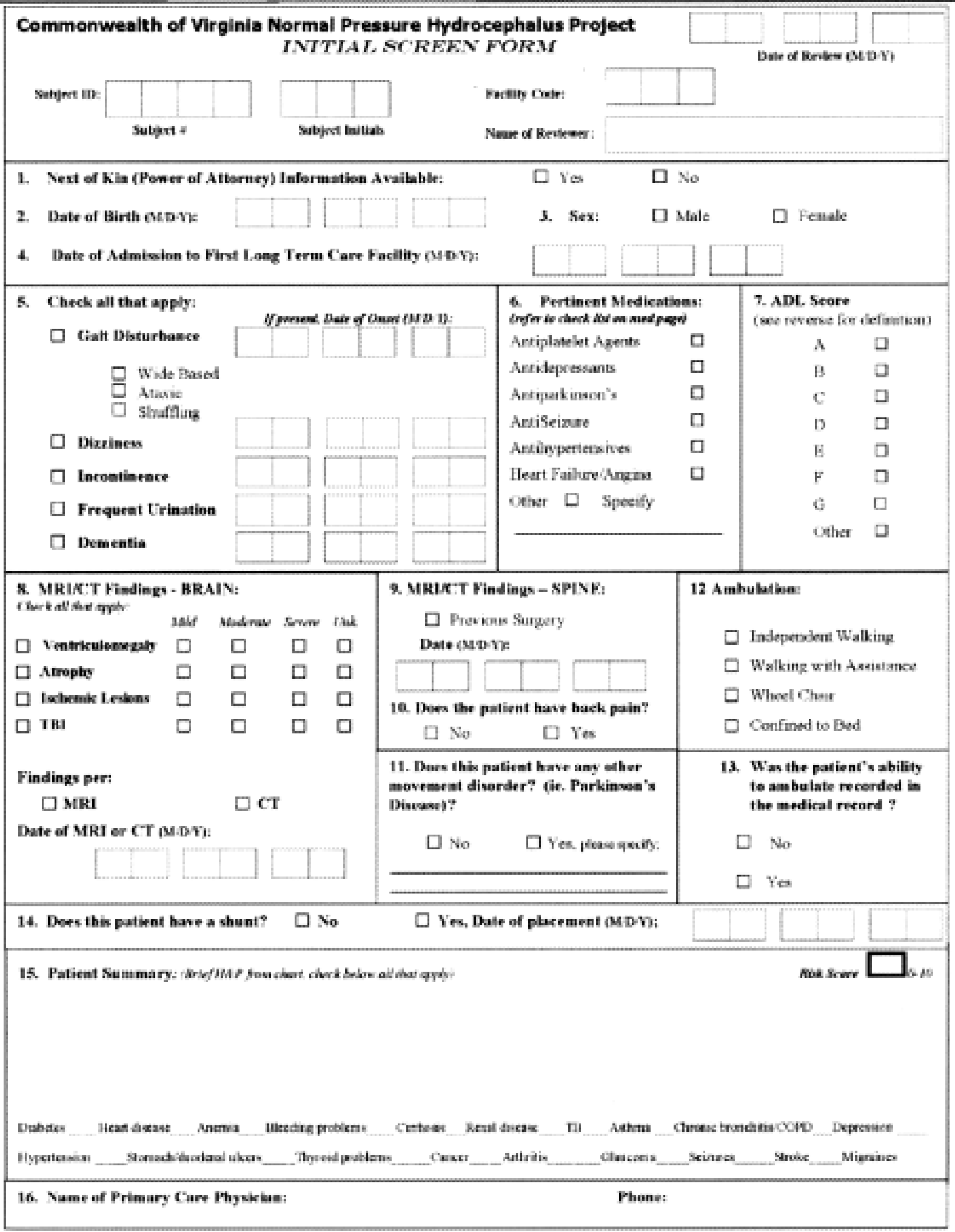

FIG. 1. Image showing the Commonwealth of Virginia Normal Pressure Hydrocephalus Project Initial Screen Form.

known origin for idiopathic NPH, and it is typically diagnosed in patients during their sixth or seventh decade of life, ${ }^{14,26}$ which suggests that age is a risk factor. ${ }^{6}$ Although the pathological mechanisms of idiopathic NPH are not fully understood, it is recognized as a disorder of CSF flow and absorption rather than formation. ${ }^{11}$ In fact, CSF flow can be disturbed by increased resistance to outflow, requiring a slightly higher pressure within the ventricles to 
maintain the production and balance of CSF; however, a disturbance in CSF circulation is not always indicative of idiopathic NPH. ${ }^{20}$ It has been postulated that a deficit in CSF absorption can cause dilation of the cerebral ventricles, but how this event leads to idiopathic NPH is not known. ${ }^{14}$ Data from studies in both animals and humans have shown that increased intraventricular CSF pressure caused by impaired CSF flow can result in ventricular enlargement. ${ }^{26}$ However, as the discrepancy between ventricular and convexity CSF pressures dissipates, episodic elevations in intraventricular CSF pressures appear to remain. Other mechanisms attributed to the development of idiopathic NPH include a reduction in blood flow and metabolism, an increase in transmantle pressure, ${ }^{14}$ and ischemic changes in white matter, altering the viscoelastic properties of brain parenchyma. ${ }^{21}$

Although the pathophysiology of idiopathic NPH remains elusive, it is one of the few dementias considered treatable and reversible. The use of shunts to reduce intraventricular volume and pressure has demonstrated promising results in patients with idiopathic NPH if the shunts are placed before the onset of cognitive deterioration. ${ }^{12,21,23}$ Despite the numerous studies focused on the utility of surgical shunts in the treatment of this disorder, their placement is controversial because of the high rate of associated complications (30-40\%)., ${ }^{5,21}$ Nonetheless, authors of several studies have demonstrated that the predominant symptoms of idiopathic NPH (gait disturbance, incontinence, and dementia) improve following the surgical placement of shunts. ${ }^{1,3,23,24}$

A lack of consensus remains regarding patient selection for optimal benefit from shunting. In a study of 18 patients with a diagnosis of idiopathic NPH, the clinical onset of the disease marked by dementia was predictive of an unfavorable outcome when assessing candidacy for shunting, whereas gait disturbance (either with or without urinary incontinence) correlated positively with functional recovery. ${ }^{5}$ Thus, patients treated in the early stages of symptom onset appear to have better outcomes from shunt surgery than patients who are diagnosed and treated in later stages of the disease.

The primary objective of this study was to estimate the prevalence of idiopathic NPH (diagnosed and undiagnosed) among residents of assisted-living and extendedcare nursing homes in southeast Virginia by using a practical screening tool. A second goal was to determine the outcome of surgical management in a subset of residents in healthcare facilities who had met the criteria for suspected idiopathic NPH.

\section{CLINICAL MATERIAL AND METHODS}

A retrospective chart-review analysis was performed using medical records from four nursing homes (two assisted-living facilities and two extended-care facilities), in accordance with the rules and regulations of the internal review board. Two hundred fifty-four patient charts were screened by a trained nurse, who traveled to each site in coordination with the site staff. Patients 85 years or older were excluded from the study. The attendant nurse at each facility created a $\log$ that identified each medical record by a number, thus maintaining anonymity and patient confidentiality. The goal of the chart review was to identify patients who matched the risk profile for idiopathic NPH by using the proposed Commonwealth of Virginia Normal Pressure Hydrocephalus Project's Initial Screen Form (Fig. 1) and to assign them a risk grade ranging from 0 (no risk) to 6 (maximum risk), as specified by the screening protocol. The form screened for the presence of idiopathic NPH symptoms documented in the medical record, including gait disturbance, incontinence or frequent urination, and dementia. The highest risk was assigned to patients who had the complete triad of symptoms. Moderate risk was assigned to those with moderate gait disturbance and one other symptom. Mild risk was assigned to patients with only mild gait disturbance or dizziness and no other symptoms. These assessments were based solely on the medical record because the protocol did not permit direct examination of the patients. Collected data were entered into a centralized database for subsequent statistical analysis revealing the estimated number of potential patients with idiopathic NPH.

Each patient's level of independence in performing various daily tasks and activities was rated using the standardized and widely accepted ADL Scale. ${ }^{8,15}$ Possible ADL scores ranged from $A$ to $G$ and were defined as follows: A, independent in feeding, continence, transferring, toileting, dressing, and bathing; $\mathrm{B}$, independent in all but one of the previously mentioned activities; $\mathrm{C}$, independent in bathing and one additional function; $\mathrm{D}$, independent in all activities except bathing, dressing, and one additional function; E, independent in all activities except bathing, dressing, toileting, and one additional function; F, independent in all activities except bathing, dressing, toileting, transferring, and one additional function; and G, dependent in all six functions.

At the beginning of the study, all patients were screened; however, as the investigators became more familiar with the profound comorbidity in these patients, it was determined that those who were 85 years or older would be excluded because of the negative risk/benefit ratio associated with treatment intervention among that cohort. Of the 254 patients screened, 107 (42\%) were excluded from study participation because of this age criterion, leaving a total of 147 eligible patients (58\%). Of these 147 patients, 94 resided in assisted-living homes and 53 in extendedcare facilities. Most of the patients were female (117 patients $[79.6 \%]$ ), and the mean age was $75.5 \pm 10.2$ years. Age data for screened patients are shown in Fig. 2.

Seventeen patients residing in healthcare facilities elected to undergo diagnostic in-hospital procedures for the evaluation and surgical management of idiopathic $\mathrm{NPH}$. After obtaining informed consent for the diagnostic procedures, CT or MR imaging, infusion studies, and/or ELD were conducted. ${ }^{17}$ Patients considered likely to be shunt responders were surgically treated with a programmable shunt (Medos Hakim shunt, Codman, Johnson \& Johnson).

\section{RESULTS}

\section{The Retrospective NPH Survey}

Symptoms of NPH. Twenty-eight (21.2\%) of 132 patients in whom gait data were available met the criteria for gait disturbance, a symptom that is almost always present 


\section{A. Marmarou, H. F. Young, and G. A. Aygok}

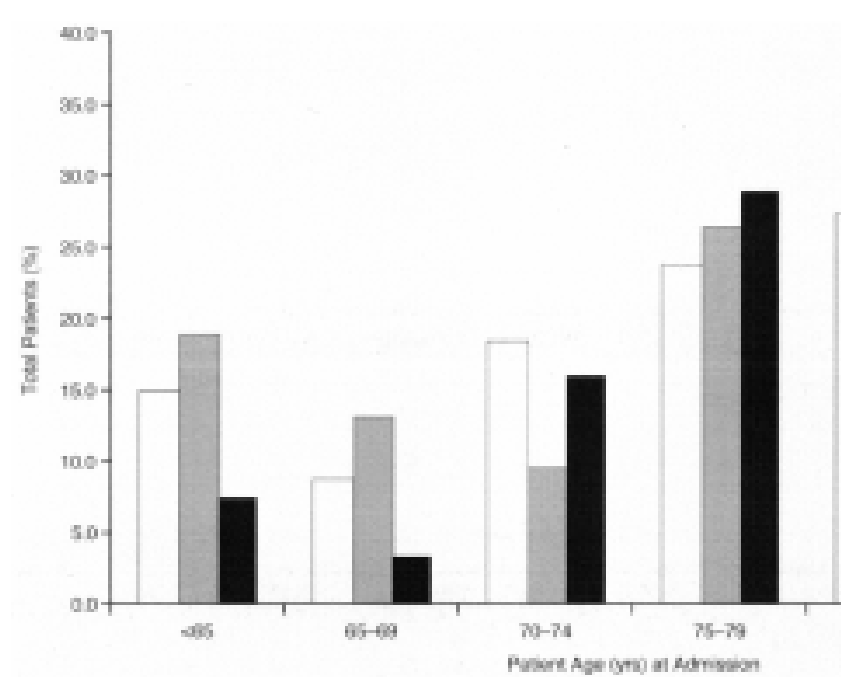

FIG. 2. Bar graph demonstrating age breakdown of screened patients with NPH who had been admitted to nursing facilities. N $=$ number of patients.

in idiopathic NPH (Fig. 3). The proportion of patients with gait disturbance increased to $23.7 \%$ (31 of 131 patients) when the criteria were widened to include dizziness. Overall, $14.7 \%$ of patients (19 of 129) had symptoms of gait disturbance and incontinence, and 9.4\% (12 of 127 patients) had symptoms of gait disturbance and dementia. Eleven $(7.5 \%)$ of 147 patients had the complete idiopathic $\mathrm{NPH}$ triad (that is, gait disturbance, incontinence, and dementia). Thus, assuming that gait disturbance plus either incontinence or dementia (or both) is necessary for the clinical diagnosis of idiopathic NPH, the overall prevalence of the disease ranged from approximately 9 to $14 \%$.

Symptoms in Assisted-Living Compared With ExtendedCare Residents. Approximately $96 \%$ of patients in each study population (assisted living and extended care) were 85 years old or younger, ages generally deemed acceptable for surgical intervention for idiopathic NPH. However, the prevalence of individual or combined elements of idiopathic NPH differed between these two populations. The greatest observed difference was the proportion of residents presenting with the complete symptom triad, which was $1.3 \%$ of those (one of 75 patients) in the assisted-living facilities and $19.2 \%$ of those (10 of 52 patients) in the extended-care centers. Respective differences were also observed in the proportion of residents with gait disturbance alone (17.7\% compared with $26.4 \%$ ), gait disturbance or dizziness (19.0\% compared with $30.8 \%$ ), gait disturbance plus incontinence $(6.5 \%$ compared with $26.9 \%)$, and gait disturbance plus dementia (1.3\% compared with $21.2 \%$ ). We also noted a greater preponderance of women in the extended-care population $(83.0 \%$ compared with $77.6 \%$ ).

Comorbidity Among Senior Residents in Assisted-Living and Extended-Care Facilities. Several residents included in the study had presented with multiple medical problems that were coincident with the suspected idiopathic NPH. In addition to the dementia associated with idiopathic NPH $(76.0 \%$ short-term memory loss and $74.5 \%$ longterm memory loss) in assisted-living and extended-care

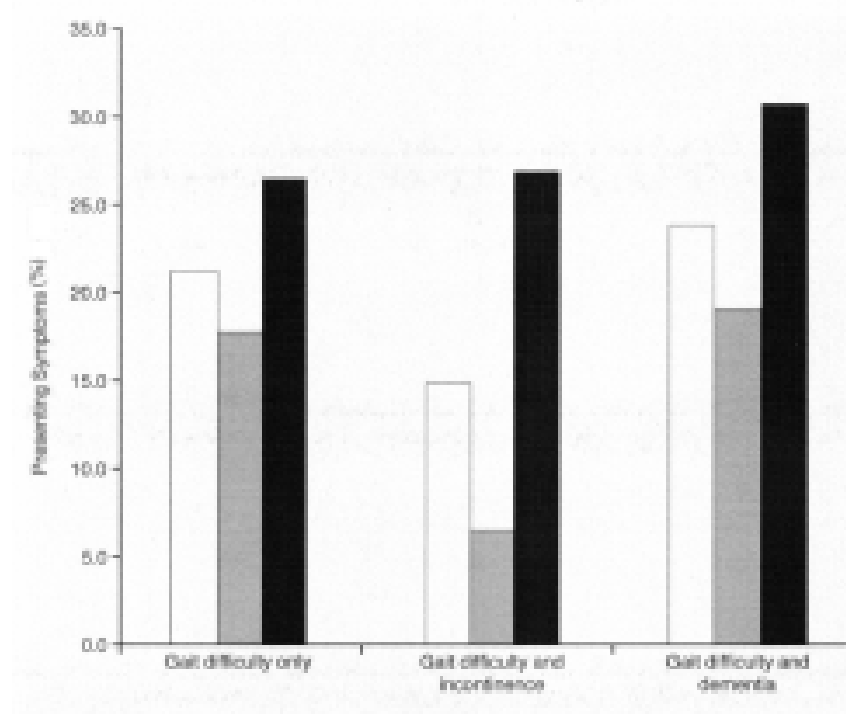

FIG. 3. Bar graph showing components of NPH in patients admitted to nursing homes in Virginia. $\mathrm{N}=$ number of patients.

residents (72.0\% compared with $62.7 \%$, respectively), depression was reported in 50.5 and $52.8 \%$ of the residents in assisted-living and extended-care facilities, respectively. Moreover, hypertension (29.7-52.8\%), diabetes $(22.5-32.7 \%)$, and heart disease $(15.7-32.7 \%)$ were highly prevalent. Overall, comorbidity was more prevalent in the residents of extended-care facilities.

Comparison of ADL Scores in Assisted-Living and Extended-Care Residents. Perhaps the most revealing variable in our findings was the measurement of ADL. The proportion of assisted-living residents who were dependent in all six functions was $30.4 \%$, compared with $62.2 \%$ of extended-care residents. As expected, no extended-care residents were completely independent in feeding, continence, transferring, toileting, dressing, and bathing, whereas $9.8 \%$ of the assisted-living residents were fully independent in these areas.

\section{Prospective Study of Idiopathic NPH Diagnostic Procedures and Treatment}

Patient Population and Symptoms. After obtaining informed consent, 17 patients fulfilling the criteria for suspected idiopathic NPH based on the survey form with a mean age of $79.5 \pm 7$ years (range 64-93 years) were admitted for diagnostic workup. Ten patients were women and seven were men. Symptoms on presentation are listed in Table 1. Of the 17 patients, 10 were wheelchair bound, six used a walker for ambulation, and one used a cane. Memory was impaired in all patients. Frank incontinence of urine was present in $70.6 \%$ (12 of 17 patients), frequent urination in 29\% (five of 17 patients), and urinary and fecal incontinence in 12\% (two of 17 patients). Among the 17 patients, Alzheimer disease had been previously diagnosed in four and Parkinson disease in three.

Diagnostic Management. Ventriculomegaly (frontal horn index $>0.3$ ) in greater proportion to atrophy was present on CT or MR images obtained in all 17 patients. A spinal tap was conducted for measurement of lumbar pressure 
TABLE 1

Summary of characteristics in 17 patients with idiopathic NPH

\begin{tabular}{|c|c|c|c|c|c|}
\hline \multirow[b]{2}{*}{$\begin{array}{l}\text { Case } \\
\text { No. }\end{array}$} & \multirow[b]{2}{*}{$\begin{array}{c}\text { Age } \\
\text { (yrs.), Sex }\end{array}$} & \multicolumn{2}{|c|}{ Symptoms* } & \multicolumn{2}{|c|}{ Comorbidity } \\
\hline & & $\begin{array}{c}\text { Gait } \\
\text { Disturbance }\end{array}$ & Incontinence & $\begin{array}{l}\text { Alzheimer } \\
\text { Disease }\end{array}$ & $\begin{array}{c}\text { Parkinson } \\
\text { Disease }\end{array}$ \\
\hline 1 & $86, \mathrm{M}$ & wheelchair & total urinary & & \\
\hline 2 & $81, \mathrm{M}$ & wheelchair & total urinary & & \\
\hline 3 & $79, \mathrm{~F}$ & wheelchair & frequency & & \\
\hline 4 & $77, \mathrm{~F}$ & wheelchair & $\begin{array}{l}\text { total urinary } \\
\quad \& \text { fecal }\end{array}$ & & present \\
\hline 5 & $78, \mathrm{~F}$ & walker & total urinary & & present \\
\hline 6 & $70, \mathrm{M}$ & walker & frequency & & \\
\hline 7 & $89, \mathrm{~F}$ & wheelchair & $\begin{array}{l}\text { total urinary } \\
\text { \& fecal }\end{array}$ & & \\
\hline 8 & $77, \mathrm{~F}$ & walker & frequency & & present \\
\hline 9 & $80, \mathrm{M}$ & wheelchair & total urinary & present & \\
\hline 10 & $74, \mathrm{~F}$ & walker & total urinary & & \\
\hline 11 & $71, \mathrm{~F}$ & walker & total urinary & & \\
\hline 12 & $93, \mathrm{M}$ & wheelchair & total urinary & & \\
\hline 13 & $85, \mathrm{M}$ & cane & frequency & & \\
\hline 14 & $85, \mathrm{M}$ & walker & frequency & & \\
\hline 15 & $81, \mathrm{~F}$ & wheelchair & total urinary & present & \\
\hline 16 & $64, \mathrm{~F}$ & wheelchair & total urinary & present & \\
\hline 17 & $82, \mathrm{~F}$ & wheelchair & total urinary & present & \\
\hline
\end{tabular}

* All patients had impaired memory.

followed by infusion studies in 12 of the 17 patients in an outpatient setting (Table 2). The mean opening pressure was $10.75 \pm 4.2 \mathrm{~mm} \mathrm{Hg}$. The mean PVI equaled $20.1 \pm$ $9.7 \mathrm{ml}$, which was less than the accepted normal level of $25 \mathrm{ml}$ and thus signified reduced brain compliance in this subset of patients. The mean CSF outflow resistance was $11.2 \pm 9.9 \mathrm{~mm} \mathrm{Hg} / \mathrm{ml} / \mathrm{min}$. This resistance was close to three times the threshold, indicating a marked absorptive deficit. ${ }^{17}$ Twelve of the 17 patients were admitted to the hospital for 3 days of ELD. Among these 12 patients, gait was improved in three, eight showed no improvement, and one patient was worse after ELD.

Response to Shunt Insertion on Follow-Up Evaluation. Eleven of the 17 patients had received shunts (Table 3). Of these 11, seven experienced improvement in gait after shunt placement. Of these seven, four had transient improvement and by 6 months returned to baseline function; three patients had retained their improvements at 1 year posttreatment. Two patients were worse at the 1-year follow up. Among the seven patients with gait improvement, four also had improved memory and five improved in incontinence. Patients whose gait outcome was worse also demonstrated worsened memory and incontinence. Hygromas developed in five of the 11 patients who had received shunts; four were treated by adjusting the programmable valve to a higher pressure, and all resolved. A hygroma in one patient required a twist-drill craniotomy but then resolved. The remaining six patients of the $17 \mathrm{did}$ not receive shunts. At 1 year posttreatment, two patients had died of sepsis related to urinary tract infection and the condition of the remaining four patients was unchanged from the initial assessment.

\section{DISCUSSION}

The findings of our survey of 147 assisted-living and extended-care residents suggest that approximately 9 to
TABLE 2

Diagnostic management in 17 patients with idiopathic $\mathrm{NPH}^{*}$

\begin{tabular}{ccccl}
\hline \hline & \multicolumn{3}{c}{ Infusion Study } & \\
\cline { 2 - 4 } Case No. & OP & $\mathrm{R}_{\text {out }}$ & PVI & ELD Outcome \\
\hline 1 & 5 & 9 & 15 & ND \\
2 & $\mathrm{ND}$ & $\mathrm{ND}$ & $\mathrm{ND}$ & worse \\
3 & $\mathrm{ND}$ & $\mathrm{ND}$ & $\mathrm{ND}$ & improved \\
4 & 15 & 10 & 24 & ND \\
5 & 14 & 12 & 18 & no change \\
6 & $\mathrm{ND}$ & $\mathrm{ND}$ & $\mathrm{ND}$ & improved \\
7 & 18 & 5 & 21 & no change \\
8 & 8 & 40 & 10 & ND \\
9 & $\mathrm{ND}$ & $\mathrm{ND}$ & $\mathrm{ND}$ & improved \\
10 & 7 & 7 & 22 & no change \\
11 & 10 & 14 & 11 & ND \\
12 & $\mathrm{ND}$ & $\mathrm{ND}$ & $\mathrm{ND}$ & no change \\
13 & 7 & 2 & 24 & no change \\
14 & 6 & 7 & 14 & no change \\
15 & 15 & 16 & 19 & ND \\
16 & 12 & 8 & 16 & no change \\
17 & 12 & 4 & 40 & no change \\
\hline$*$ & ND $=$ test not done; OP $=$ opening pressure. & \\
& & &
\end{tabular}

$14 \%$ of patients are at risk for or may suffer from idiopathic NPH. Note, however, that of the 147 patients included in the survey, only five had undergone MR imaging or CT, the established means of diagnosing idiopathic $\mathrm{NPH} .{ }^{12}$ This discrepancy suggests that idiopathic NPH symptoms often are overlooked or attributed to other causes. It is plausible that on reevaluation, several study participants may have benefited from surgical shunt insertion if a proper diagnosis had been made and the disease had been treated in its early stages.

Survey data also suggest that assumptions of poor surgical candidacy (standardized age criteria) or premature judgments of surgical benefit (other comorbidities that might decrease benefit) should not exclude extended-care patients from undergoing thorough diagnostic evaluations. In a recent study in which supplementary tests were used to identify shunt-responsive patients, improvement following 3-day drainage was found to be independent of age. ${ }^{17}$ Moreover, $96 \%$ of the extended-care population in the present study was younger than 85 years of age (suitable age for surgical intervention), and nearly $40 \%$ demonstrated at least some degree of independence (based on ADL criteria). The idiopathic NPH symptom triad was present in nearly one fifth of the extended-care residents included in this analysis.

As expected, comorbidity was higher in the extendedcare population-residents with more problems would be expected to require a greater amount of care. It is widely recognized that patients with idiopathic NPH frequently have a variety of comorbidities, including Alzheimer disease and cerebrovascular disease. ${ }^{2}$ Moreover, it is not unexpected to observe signs of depression, confusion, intellectual impairment, or gait difficulties in the elderly population because these are part of the normal aging process. ${ }^{13}$ Unfortunately, the presence of other diseases or conditions that mimic symptoms of idiopathic NPH can confound or delay diagnosis and intervention. Because surgical intervention provides the greatest benefit when performed early after the onset of idiopathic $\mathrm{NPH},{ }^{18}$ 
A. Marmarou, H. F. Young, and G. A. Aygok

TABLE 3

Treatment details in 17 patients with idiopathic NPH

\begin{tabular}{clll}
\hline \hline Case No. & Shunt & \multicolumn{1}{c}{ Status at 1 Yr Follow-Up } & Shunt Complication \\
\hline 1 & yes & no change & hygroma \\
2 & yes & transient moderate improvement & hygroma \\
3 & yes & permanent excellent improvement & hygroma \\
4 & yes & transient mild improvement & hygroma \\
5 & yes & transient moderate improvement & complete \\
6 & yes & worse & partial \\
7 & yes & no change & complete \\
8 & yes & permanent moderate improvement & \\
9 & yes & permanent moderate improvement & \\
10 & yes & worse & \\
11 & yes & transient mild improvement & \\
12 & no & no change & death \\
13 & no & no change & \\
14 & no & no change & \\
15 & no & no change & \\
17 & & death &
\end{tabular}

reports of no response to shunt placement must be interpreted carefully. Such reports may be falsely elevated because the early signs of idiopathic NPH may have been overlooked and interventions performed too late. ${ }^{2}$ Thus, special attention must be given to patients who have multiple medical problems that could possibly confound making an accurate diagnosis of idiopathic NPH and consequently reduce the likelihood of a successful treatment outcome. ${ }^{16}$

In recent years, there has been growing emphasis on the importance of health-related quality of life among patient populations. In a study in which they compared the quality of life among patient cohorts with CSF disorders, Gelling and colleagues ${ }^{10}$ found that scores on the 36-Item Short Form Health Survey and the Mini-Mental State Examination ${ }^{4,9,25}$ were significantly lower in patients with idiopathic NPH than in those with other CSF disorders. They argued that the scores reflected the need for assessment scales that more reliably capture the data in patients with cognitive deterioration, who might not be able to understand or complete such scales. These findings also emphasize the need for early diagnosis and treatment in those at high risk for idiopathic NPH, before other medical problems become so overwhelming that patients who might have benefited become refractory to treatment. In a study of cerebrospinal shunt insertion for the treatment of idiopathic NPH, 25 (58.1\%) of 43 patients scored less than 24 on the Mini-Mental State Examination before surgical treatment. Six months after surgery, however, seven of these patients had scores within the normal range. ${ }^{21}$ The ADL Scale was also applied in these study participants; 19 patients $(44.2 \%)$ were considered independent in their ADL, nine $(20.9 \%)$ required some help, and 15 (34.9\%) were considered totally dependent before surgery. However, 6 months after surgery, 33 patients $(76.7 \%)$ were able to cope with daily activities, four $(9.3 \%)$ were partially dependent, and only six (14\%) remained completely dependent.

Although the present study was a retrospective chart analysis based on a small population that is representative of a southeast nursing facility, the results reinforce the need for future investigations of nursing-care facilities globally to ascertain the percentage of patients whose disease is misdiagnosed or undiagnosed and thus who are not receiving the necessary treatment for what is a potentially reversible syndrome if caught early. A cost-benefit analysis is needed to evaluate the overall benefit. Of course, full-time nursing care is expensive and over time would exceed the costs of screening and surgical intervention.

A limitation of the present survey is its retrospective nature, and thus it was impossible to definitively diagnose the disease (CT scanning is required) and treat patients surgically to assess response. The in-hospital study of 17 patients living in healthcare facilities at the time of assessment presented us with an opportunity to evaluate a sample population prospectively. All patients seen at the clinic had presented with the classic symptom triad of gait disturbance, incontinence, and dementia. In fact, most were wheelchair bound, which is common in patients who are at risk for a fall or who cannot ambulate. We found that all patients screened in this population had ventriculomegaly, completing the diagnostic symptomology for idiopathic NPH. Supplementary test data revealed relatively normal opening pressures, poor CSF absorption indicated by high outflow resistance $\left(\mathrm{R}_{\text {out }}\right)$, and low PVI indicating reduced compliance or tight brains. The outcome of shunt insertion based on supplementary tests to identify shunt responders revealed only seven of 11 patients showing some improvement, which is consistent with findings in previous studies. ${ }^{17}$ Note, however, that this improvement was not sustained in four of the seven patients. One explanation for this poor response is the fact that most of the patients had been wheelchair bound for some years, suggesting that they had reached a stage beyond the therapeutic window. Nonetheless, three of the patients who had been in wheelchairs were able to walk, and this improvement was sustained at 1 year posttreatment. Considering all of these findings, we must conclude that the current supplementary tests remain predictive of a positive response to shunt insertion but cannot indicate whether a favorable outcome will be sustained in this patient population, most of whom have been confined to a 
wheelchair for a prolonged period of time. The data reemphasize the notion of a finite window of opportunity for managing idiopathic $\mathrm{NPH}$ - early diagnosis and treatment are imperative.

\section{CONCLUSIONS}

It is important that a practical screening tool specific to the signs and symptoms of idiopathic NPH be applied before patients are admitted to nursing-care facilities. This tool could take the form of a one-page query of symptoms specific to the disease, which would include the presence of one or more of the classic symptoms, that is, gait disturbance, incontinence, and dementia. Recently, it has been proposed that the diagnosis of idiopathic NPH be subdivided into probable, possible, and unlikely. ${ }^{22}$ The elements required for this classification should be incorporated into the screening tool. Excluding other pathophysiology, physicians could implement neuroimaging to determine the presence of ventricle enlargement consistent with idiopathic NPH. If the results of both the screening and neuroimaging studies validate a diagnosis of idiopathic $\mathrm{NPH}$, patients could be referred to specialists to determine surgical candidacy, thus potentially providing such patients with an improved quality of life and reducing their need for specialized housing and care. Our experience with the in-hospital assessment of nonambulatory patients living in healthcare facilities suggests that a standardized protocol for diagnosis and supplementary tests to identify shunt responders must be used as early as possible.

\section{Acknowledgments}

We thank the Virginia State Legislature for its support and Frank and Orinna Hargrove for their encouragement.

\section{References}

1. Anderson RC, Grant JJ, de la Paz R, Frucht S, Goodman RR: Volumetric measurements in the detection of reduced ventricular volume in patients with normal-pressure hydrocephalus whose clinical condition improved after ventriculoperitoneal shunt placement. J Neurosurg 97:73-79, 2002

2. Bech-Azeddine R, Waldemar G, Knudsen GM, Hogh P, Bruhn P, Wildschiodtz $G$, et al: Idiopathic normal-pressure hydrocephalus: evaluation and findings in a multidisciplinary memory clinic. Eur J Neurol 8:601-611, 2001

3. Benzel EC, Pelletier AL, Levy PG: Communicating hydrocephalus in adults: prediction of outcome after ventricular shunting procedures. Neurosurgery 26:655-660, 1990

4. Bowling AC, Stewart TM: Current complementary and alternative therapies for multiple sclerosis. Curr Treat Options Neurol 5:55-68, 2003

5. Caruso R, Cervoni L, Vitale AM, Salvati M: Idiopathic normalpressure hydrocephalus in adults: result of shunting correlated with clinical findings in 18 patients and review of the literature. Neurosurg Rev 20:104-107, 1997

6. Casmiro M, Cacciatore FM, D’Alessandro R: The pathogenesis of idiopathic normal pressure hydrocephalus: an open problem. Funct Neurol 4:403-410, 1989

7. Dippel DW, Habbema JD: Probabilistic diagnosis of normal pressure hydrocephalus and other treatable cerebral lesions in dementia. J Neurol Sci 119:123-133, 1993

8. Fillenbaum GG, Heyman A, Huber MS, Ganguli M, Unverzagt FW: Performance of elderly African American and White community residents on the CERAD Neuropsychological Battery. J Int Neuropsychol Soc 7:502-509, 2001
9. Folstein MF, Folstein SE, McHugh PR: "Mini-mental state". A practical method for grading the cognitive state of patients for the clinician. J Psychiatr Res 12:189-198, 1975

10. Gelling L, Iddon J, McVicar A, Pickard JD: CSF circulation disorders: measuring progress in patients through quality of life and hope. J Clin Nurs 13:589-600, 2004

11. Gleason PL, Black PM, Matsumae M: The neurobiology of normal pressure hydrocephalus. Neurosurg Clin N Am 4:667675, 1993

12. Hakim CA, Hakim R, Hakim S: Normal-pressure hydrocephalus. Neurosurg Clin N Am 36:761-773, 2001

13. Hakim S, Adams RD: The special clinical problem of symptomatic hydrocephalus with normal cerebrospinal fluid pressure. Observations on cerebrospinal fluid hydrodynamics. J Neurol Sci 2:307-327, 1965

14. Hebb AO, Cusimano MD: Idiopathic normal pressure hydrocephalus: a systematic review of diagnosis and outcome. Neurosurgery 49:1166-1184, 2001

15. Katz S, Ford AB, Moskowitz RW, Jackson BA, Jaffe MW: Studies of illness in the aged. The index of ADL: a standardized measure of biological and psychosocial function. JAMA 185: 914-919, 1963

16. Marmarou A, Bergsneider M, Relkin N, Klinge P, Black PM: Development of guidelines for idiopathic normal-pressure hydrocephalus: introduction. Neurosurgery 57 (3 Suppl): S1-S3, 2005

17. Marmarou A, Young HF, Aygok GA, Sawauchi S, Tsuji O, Yamamoto T, et al: Diagnosis and management of idiopathic normal-pressure hydrocephalus: a prospective study in $151 \mathrm{pa}-$ tients. J Neurosurg 102:987-997, 2005

18. Meier U, Konig A, Miethke C: Predictors of outcome in patients with normal-pressure hydrocephalus. Eur Neurol 51:59-67, 2004

19. Mori K: Management of idiopathic normal-pressure hydrocephalus: a multiinstitutional study conducted in Japan. J Neurosurg 95:970-973, 2001

20. Owler BK, Pickard JD: Normal pressure hydrocephalus and cerebral blood flow: a review. Acta Neurol Scand 104: 325-342, 2001

21. Poca MA, Mataro M, del Mar Matarin M, Arikan F, Junque C, Sahuquillo J: Is the placement of shunts in patients with idiopathic normal-pressure hydrocephalus worth the risk? Results of a study based on continuous monitoring of intracranial pressure. J Neurosurg 100:855-866, 2004

22. Relkin N, Marmarou A, Klinge P, Bergsneider M, Black PM: Diagnosing idiopathic normal-pressure hydrocephalus. Neurosurgery 57 (3 Suppl): S4-S16, 2005

23. Savolainen S, Hurskainen H, Paljarvi L, Alafuzoff I, Vapalahti M: Five-year outcome of normal pressure hydrocephalus with or without a shunt: predictive value of the clinical signs, neuropsychological evaluation and infusion test. Acta Neurochir (Wien) 144:515-523, 2002

24. Stambrook M, Cardoso E, Hawryluk GA, Eirikson P, Piatek D, Sicz G: Neuropsychological changes following the neurosurgical treatment of normal pressure hydrocephalus. Arch Clin Neuropsychol 3:323-330, 1988

25. Tombaugh TN, McIntyre NJ: The mini-mental state examination: a comprehensive review. J Am Geriatr Soc 40:922-935, 1992

26. Vanneste JA: Diagnosis and management of normal-pressure hydrocephalus. J Neurol 247:5-14, 2000

Manuscript submitted February 2, 2007.

Accepted in final form March 2, 2007.

This work was supported in part by the Virginia State Legislature.

Address reprint requests to: Anthony Marmarou, Ph.D., Division of Neurosurgery, Virginia Commonwealth University, P.O. Box 980508, Richmond, Virginia 23298-0508. email: marmarou@ hsc. vcu.edu. 
Author Names, et al. 